\title{
Comparison of efficacy between video-assisted thoracoscopic surgery and thoracotomy in children with mediastinal tumors: 6-year experience
}

\author{
Min Da", Wei Peng", Xuming Mo", Ming Fan, Kaihong Wu, Jian Sun, Jirong Qi, Yuxi Zhang \\ Department of Cardiothoracic Surgery, Children's Hospital of Nanjing Medical University, Nanjing 210008, China \\ Contributions: (I) Conception and design: X Mo, W Peng; (II) Administrative support: None; (III) Provision of study materials or patients: M Da; (IV) \\ Collection and assembly of data: M Fan; (V) Data analysis and interpretation: M Fan; (VI) Manuscript writing: All authors; (VII) Final approval of \\ manuscript: All authors. \\ \#These authors equally contributed to this work. \\ Correspondence to: Wei Peng; Xuming Mo. Department of Cardiothoracic Surgery, Children's Hospital of Nanjing Medical University, Jiangdong \\ South No. 8 Road, Nanjing 210008, China. Email: pwking@sina.com; mohsuming15@sina.com.
}

Background: Video-assisted thoracic surgery (VATS) has been increasingly used in pediatric patients. We evaluated the outcomes of VATS and thoracotomy for pediatric mediastinal tumors.

Methods: A total of 137 patients who underwent surgery for mediastinal tumors at our department from March 2012 to September 2018 were recruited in this retrospective study. Forty-three patients were treated by VATS and ninety-four underwent thoracotomy (two patients who underwent open surgery were excluded from the study because they were lost to follow-up). Data including demographic information, tumor characteristics, operative time, conversion of surgery, blood transfusion, postoperative hospital stay, mortality and recurrence were collected and compared between the two groups.

Results: No significant difference was found in gender, age, weight and tumor distribution between the two groups. The number of patients with malignant tumors who underwent thoracotomy was significantly higher than those who received VATS $(78.0 \%$ vs. $22.0 \%, \mathrm{P}=0.04)$. Patients received thoracotomy had significantly larger mean tumor diameter than those who underwent VATS (7.6 \pm 3.8 vs. $4.4 \pm 1.7 \mathrm{~cm}, \mathrm{P}<0.001)$. The intraoperative transfusion rate in the thoracotomy group was significantly higher than that in the VATS group (67.4\% vs. $14.0 \%, \mathrm{P}<0.001)$, so was the amount of blood transfusion $(148.1 \pm 150.7$ vs. $23.3 \pm 61.1 \mathrm{~mL}$, $\mathrm{P}<0.001)$. The VATS group had significantly shorter operative time as compared with the thoracotomy group (94.3 \pm 40.9 vs. $133.5 \pm 72.1 \mathrm{~min}, \mathrm{P}=0.002)$. During follow-up, local recurrence was found in $8(8.7 \%)$ patients who underwent thoracotomy, and no significant difference was found in local recurrence rate between the two groups. Four patients who underwent thoracotomy died, and no significant difference was found in mortality between two groups.

Conclusions: Due to less blood transfusion, shorter operative time and postoperative hospital stay, VATS is a safer surgical treatment for pediatric mediastinal tumors than thoracotomy.

Keywords: Video-assisted thoracic surgery (VATS); thoracotomy; children; mediastinal tumors

Submitted Jun 25, 2019. Accepted for publication Sep 30, 2019.

doi: $10.21037 / \mathrm{atm} .2019 .10 .81$

View this article at: http://dx.doi.org/10.21037/atm.2019.10.81 


\section{Introduction}

Recently, video-assisted thoracic surgery (VATS) has become a common surgical procedure for pediatric patients. Since the 1990s, numerous reports $(1,2)$ have evaluated the safety and feasibility of VATS in diagnosing and treating common or benign pediatric thoracic diseases. At present, though thoracotomy is often adopted for surgical resection of pediatric mediastinal lesions, the use of VATS in pediatric mediastinal surgery has begun to spread (3-5). In this retrospective study, 137 cases of pediatric mediastinal tumors treated with either thoracotomy or VATS were retrospected to determine the safety and feasibility of VATS.

\section{Methods}

\section{Study population}

The records of the patients who received surgery at our department between March 2012 and September 2018 were obtained. A total of 167 patients with a mediastinal lesion were identified. Of them, three patients who had large tumors in anterior or upper mediastinum died of respiratory collapse during general anesthesia and were excluded from our study. Clinically, two of them might have malignant lymphomas and one might have germ cell tumor (Table 1). All patients who had received puncture biopsy for diagnostic intent were not involved. A total of 137 patients who had undergone VATS or thoracotomy were enrolled in this study. The patients' age ranged from 0 to 155 months. This retrospective study was approved by the Medical Ethics Committee of Children's Hospital of Nanjing Medical University.

Data including demographic information, tumor characteristics, operative time, conversion of surgery, blood transfusion, length of postoperative hospital stay, mortality and recurrence were collected and compared between the two groups. Treatment-related complications within 30 days of surgery were also analyzed as safety assessment.

All patients were assessed with computed tomography (CT) before surgery, and magnetic resonance imaging was prescribed for those suspected of having paravertebral sulcus or vertebral invasions. Five patients' operations were performed with the assistance of a neurosurgeon because their lesions were found to be associated with the spinal canal (Table 2). Early complications were defined as undesired events during hospitalization or within the first week after discharge. Late complications were defined as unfavorable events that occurred between day 7 and day 30 after discharge. Physical examination and CT scans were performed 6 months and 12 months after surgery, and then once a year to detect recurrence.

All the operations were conducted by 3 surgeons, who determined the surgical approach according to the size and location of the tumor and their preference (Figure 1).

\section{Statistical analysis}

The data of continuous variable were skewed normal distribution. Statistical analysis was performed using Fisher's exact test (categorical variable) or the Mann-Whitney test (continuous variable) using SPSS software (version 18.0, SPSS Inc., Chicago, IL, USA). $\mathrm{P}<0.05$ was considered statistically significant.

\section{Results}

A total of 137 patients were enrolled in the study, of which $43(31.4 \%)$ underwent VATS and 94 (68.6\%) underwent thoracotomy (including 4 whose surgical approach was converted). Two patients who underwent open surgery were excluded from the study because they were lost to followup. There was no significant difference in gender, age, weight and tumor distribution between the two groups. The number of patients with malignant tumors who underwent thoracotomy was significantly higher than those who received VATS $(78.0 \%$ vs. $22.0 \%, \mathrm{P}=0.04)$. The patients who received thoracotomy had significantly larger mean tumor diameter than those who underwent VATS (7.6 \pm 3.8 vs. $4.4 \pm 1.7 \mathrm{~cm}, \mathrm{P}<0.001$ ) (Table 3).

The thoracotomy group had a significantly higher rate of intraoperative transfusion than the VATS group $(67.4 \%$ vs. $14.0 \%$ for VATS, $\mathrm{P}<0.001)$, so was the amount of blood transfusion $(148.1 \pm 150.7$ vs. $23.3 \pm 61.1 \mathrm{~mL}, \mathrm{P}<0.001)$. The VATS group had significantly shorter operative time as compared with the thoracotomy group $(94.3 \pm 40.9$ vs. $133.5 \pm 72.1 \mathrm{~min}, \mathrm{P}=0.002)$. There was no significant difference in the mean postoperative hospital stay or postoperative chemotherapy between the two groups (Table 4).

There was no difference between the two groups in terms of postoperative complications. The most common early complication was diaphragmatic eventration which occurred to 3 (2.2\%) patients. Two patients (1.5\%) had repeated pleural effusion and one had an esophageal rupture during thoracotomy for which esophageal repair 
Table 1 Patients with respiratory collapse

\begin{tabular}{|c|c|c|c|c|c|c|c|c|}
\hline Time & Patients & Gender & Age & Location & $\begin{array}{l}\text { Possible pathological } \\
\text { diagnosis }\end{array}$ & Chief complaint & Enrollment & End-event \\
\hline 2013.01 & A & Female & $13 Y$ & Upper mediastinum & Lymphoma? & $\begin{array}{l}\text { Cough and asthma with left } \\
\text { chest pain for two days }\end{array}$ & No & Death \\
\hline 2014.07 & $\mathrm{~B}$ & Male & $13 Y$ & Anterior mediastinum & Germ cell tumor? & $\begin{array}{l}\text { Chest tightness with } \\
\text { asthma for a week }\end{array}$ & No & Death \\
\hline 2015.03 & $\mathrm{D}$ & Male & $11 \mathrm{Y}$ & Anterior mediastinum & Mature cystic teratoma & $\begin{array}{l}\text { Chest tightness with } \\
\text { asthma for ten days }\end{array}$ & Yes & Survival \\
\hline
\end{tabular}

Table 2 Patients with intraspinal invasion

\begin{tabular}{|c|c|c|c|c|c|c|}
\hline Patient & Gender & Age (months) & $\begin{array}{l}\text { Preoperative } \\
\text { chemotherapy }\end{array}$ & Pathology & Recurrence & Follow-up (months) \\
\hline E & Female & 37 & $\mathrm{~N}$ & Malignant rhabdomyomas & $\mathrm{N}$ & 13 \\
\hline $\mathrm{F}$ & Female & 25 & $\mathrm{~N}$ & Ganglion cell neuroblastoma (nodular) & $\mathrm{N}$ & 36 \\
\hline G & Male & 25 & $\mathrm{~N}$ & $\begin{array}{l}\text { Ganglion cell neuroblastoma (nodular) with } \\
\text { lymph node metastasis }\end{array}$ & $\mathrm{N}$ & 16 \\
\hline I & Female & 37 & $\mathrm{~N}$ & Neuroblastoma (differentiated) & Y, reoperation & 26 \\
\hline
\end{tabular}
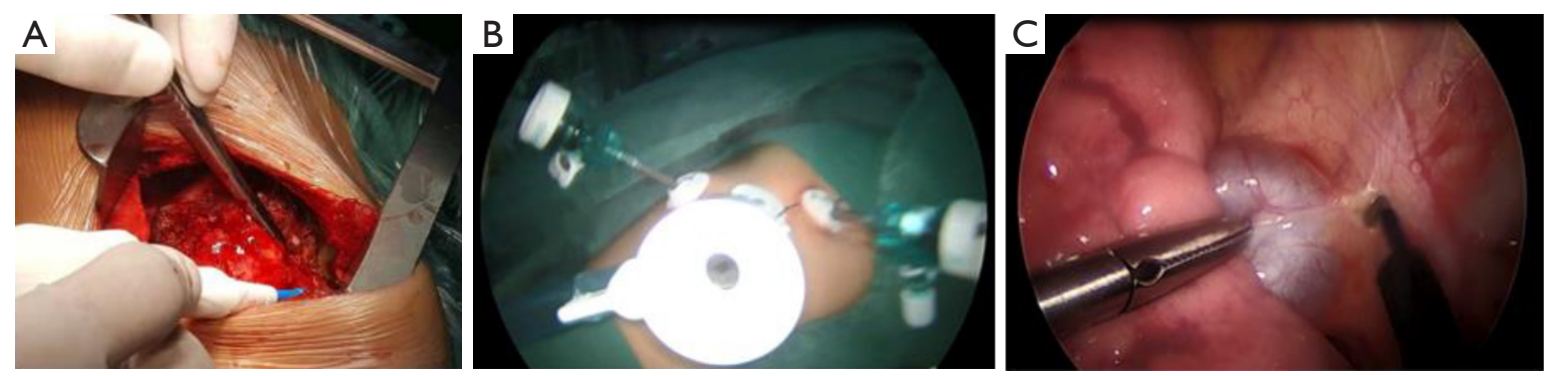

Figure 1 (A) The thoracotomy usually uses a posterolateral incision and chooses the intercostal space according to the location of the tumor. (B,C) The thoracoscope is introduced through the 3th or 7th intercostal space of the midline according to the location of the tumor. Two surgical ports are inserted through the 4th, 5 th and 6th intercostal spaces of the anterior axillary line and the 6th to 8 th intercostal spaces of the posterior axillary line to form a triangular configuration.

was performed. In the late period, two patients $(1.5 \%)$ had wound site infection.

One patient who had tumor invasion in the main airway died of airway obstruction during thoracotomy. No mortality occurred in either group in the first 30 postoperative days. The pathological data of all cases are listed in Table 5. Of all the cases, ganglion cell neuroblastoma ( $\mathrm{n}=26,19.3 \%)$ was the most common lesion, and neuroblastoma $(\mathrm{n}=22,16.3 \%)$ ranked the second. Neurogenic tumors account for $41.5 \%$ of all lesions. During the follow-up, no significant difference was found in recurrence rate between the two groups; local recurrences were found in $8(8.7 \%)$ patients who underwent open surgery. Among these patients, three (including one who refused chemotherapy) died of recurrence at month 2, 3, 8 after surgery respectively; reoperation was attempted in 
Table 3 Demographic data and tumor characteristics

\begin{tabular}{|c|c|c|c|}
\hline Variable & Thoracotomy $(n=92)$ & VATS $(n=43)$ & $P$ value \\
\hline Gender & & & 0.353 \\
\hline Male & $63.3 \%[38]$ & $36.7 \%[22]$ & \\
\hline Female & $72.0 \%[54]$ & $28.0 \%[21]$ & \\
\hline Location & & & 0.815 \\
\hline Upper mediastinum & $65.9 \%[27]$ & $34.1 \%[14]$ & \\
\hline Anterior mediastinum & $75.0 \%[24]$ & $25.0 \%[8]$ & \\
\hline Middle mediastinum & $62.5 \%[5]$ & $37.5 \%[3]$ & \\
\hline Benign & $60.5 \%[46]$ & $39.5 \%[30]$ & \\
\hline Malignancy & $78.0 \%[46]$ & $22.0 \%[13]$ & \\
\hline Size (cm) & $7.6 \pm 3.8[3-24]$ & $4.4 \pm 1.7[2.4-10]$ & $<0.001$ \\
\hline
\end{tabular}

Data are presented as mean \pm SD, \% [n], [minimum-maximum]. VATS, video-assisted thoracic surgery.

Table 4 Operative details and postoperative outcomes

\begin{tabular}{lccc}
\hline Operative details/outcomes & Thoracotomy $(\mathrm{n}=92)$ & VATS $(\mathrm{n}=43)$ & P value \\
\hline Intraoperative transfusion & $67.4 \%[62]$ & $14.0 \%[6]$ & $<.001$ \\
Amount of blood transfusion $(\mathrm{mL})$ & $148.1 \pm 150.7$ & $23.3 \pm 61.1$ & $<0.001$ \\
Operative time $(\mathrm{min})$ & $133.5 \pm 72.1$ & $94.3 \pm 40.9$ & 0.002 \\
Postoperative hospital stay $(\mathrm{d})$ & $9.8 \pm 4.6$ & $10.0 \pm 5.5$ & 0.727 \\
Postoperative chemotherapy & $27.2 \%[25]$ & $16.3 \%[7]$ & 0.195 \\
Postoperative complications & $6.5 \%[6]$ & $4.7 \%[2]$ & 0.686 \\
Recurrence & $8.7 \%[8]$ & $0 \%[0]$ & 0.056 \\
Mortality & $4.3 \%[4]$ & $0 \%[0]$ & 0.302 \\
\hline
\end{tabular}

VATS, video-assisted thoracic surgery.

two patients whose had recurrent neuroblastoma and mixed germ cell tumor at postoperative month 10 and 11.2; two patients who had recurrent ganglion cell neuroblastoma at postoperative month 12 and 19 refused reoperation because they could not afford the expenses; another patient had a recurrent lymphangioma at postoperative month 2 . The latter three patients were kept under close follow-up. Although four patients underwent thoracotomy died, there was no significant difference in mortality between the two groups (Figure 2).

\section{Discussion}

Fatalities resulting from severe airway and cardiac compression during general anesthesia induction with mediastinal tumors have been reported $(6,7)$. Kawaguchi et al. (8) have reported orthopnea as a predictive risk factor associated with airway obstruction during general anesthesia 
Table 5 Pathological data of all cases

\begin{tabular}{|c|c|c|c|}
\hline Variable & Total & Thoracotomy $(\mathrm{n}=92)$ & VATS $(n=43)$ \\
\hline Neuroblastoma & 22 & 17 & 5 \\
\hline Mature cystic teratoma & 18 & 13 & 5 \\
\hline Lymphangioma & 11 & 7 & 4 \\
\hline Ganglion neuroma & 6 & 5 & 1 \\
\hline lymphoma & 3 & 3 & 0 \\
\hline Immature teratoma & 3 & 3 & 0 \\
\hline Lymphatic hemangioma & 3 & 2 & 1 \\
\hline Thymic lipoma & 3 & 3 & 0 \\
\hline Thymic cyst & 3 & 2 & 1 \\
\hline Hemangioma & 2 & 1 & 1 \\
\hline Cardiac myxoma & 1 & 1 & 0 \\
\hline Small cell malignant tumor & 1 & 1 & 0 \\
\hline Esophageal cyst & 1 & 1 & 0 \\
\hline Embryonic rhabdomyosarcoma & 1 & 1 & 0 \\
\hline Mixed germ cell tumor & 1 & 1 & 0 \\
\hline
\end{tabular}

VATS, video-assisted thoracic surgery.

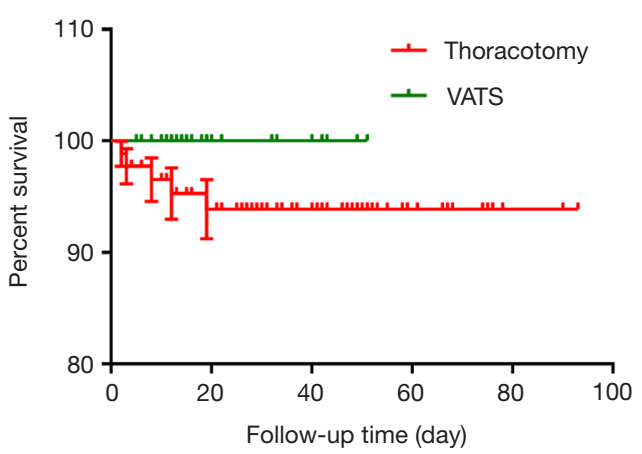

Figure 2 The overall survival plots of thoracotomy $v s$. VATS. There was no significant difference between the two groups. VATS, video-assisted thoracic surgery. induction for pediatric mediastinal tumors. In our study, the 3 patients who died during anesthesia induction developed cough, wheezing and chest tightness; two of them with orthopnea could hardly lie flat. Despite the use of the reclining position and the rigid cannula during intubation, severe airway collapse occurred. For cases with suspected lymphoma in the upper or anterior mediastinum and a high risk of cardiorespiratory morbidity during general anesthesia induction, prebiopsy steroid treatment was offered to reduce the tumor size and lower the anesthesia risk. For the cases with compression caused by a large substantial tumor, if necessary, the tumor was removed under the extracorporeal circulation via the femoral 
artery and vein cannula (9). One patient survived with this method but had more bleeding loss during surgery due to heparinization. In addition, improvements in anesthesia strategies, including induction of anesthesia in a sitting position and maintenance of spontaneous breathing, were effective in reducing the risk of anesthesia.

Mediastinal tumors have complex pathology and varied subtypes, including neurogenic tumors, reproductivederived tumors, lymphoid tumors, teratomas, bronchogenic cysts, cardiac tumors and lipomas, among which neurogenic tumors and teratomas are the most common types (10). In our study, 56 (41.5\%) of the 135 mediastinal tumors were neurogenic tumors, 21 (15.6\%) were teratoma, 19 (14.1\%) were bronchogenic cysts, and 17 (12.6\%) were vascular origin tumors. Mediastinal tumors have no obvious clinical symptoms in the early stage, but the development of tumors may stimulate the pleura and compress trachea, great vessels, esophagus and nerves. As a result, atypical clinical symptoms such as cough, respiratory distress, lip cyanosis and chest pain may occur.

Thoracotomy and VATS are two most recommended options for mediastinal lesions. The incision for thoracotomy is based on the position of the tumor. The children's chest is elliptical and elastic, which avails to easy and clear exposure of the tumor (11). Also, thoracotomy is a comparatively simple procedure and can be applied for large tumors and severe thoracic adhesion. However, open surgery may cause more bleeding, resulting in longer operative and recovering time. Moreover, thoracotomy breaks the thoracic structure, which seriously affects the patient's appearance. Thoracoscopic surgery has been reported to detect and diagnose chest diseases in pediatric patients since 1976 (12). Due to the limitations of surgical instruments and techniques, the use of thoracoscopy is relatively limited until Peter W. first introduced VATS in pediatric patients in 1993 (13). Since then, VATS surgery has become popular in children with chest diseases, including mediastinal tumors. Compared with open surgery, thoracoscopic technique has the advantages of clear anatomy, less bleeding, small incision, quick recovery, and less postoperative complications $(4,5,14)$. It has gradually become a routine procedure for pediatric thoracic surgery. But VATS has its own limitations, such as limited operating space and high requirement of the anesthesia and surgical skills.

In our study, the two groups of patients had similar the demographic data. The thoracotomy group has a higher malignancy rate, however, patients in VATS group showed better results in operative time, rate and amount of blood transfusion and postoperative hospital stay. Postoperative pathology showed no statistical difference in middle- or high-risk pathological classification for chemotherapy between the two groups. No statistically significant difference was found in the occurrence of postoperative complications between the two groups. Neither could we find statistically significant difference in either local recurrence rate or operative and postoperative mortality between the two groups. These data evidence the safety and feasibility of VATS in pediatric patients with mediastinal tumors.

Liu et al. (15) believe that open surgical methods are preferable for tumors $>8 \mathrm{~cm}$. Li et al. (16) report that thoracotomy is necessary for tumors larger than $6 \mathrm{~cm}$. While those are all based on surgical data of adults, few have been reported in children. In this study, the mean tumor size of thoracotomy group was $7.6 \pm 3.8 \mathrm{~cm}$, and VAST group was $4.4 \pm 1.7 \mathrm{~cm}(\mathrm{P}<0.001)$. A total of $46(78.0 \%)$ patients with malignant tumors underwent thoracotomy, and $13(22.0 \%)$ underwent VATS $(\mathrm{P}<0.05)$. Therefore, our results also revealed open surgery is preferable for patients with large and malignant masses.

In our study, four patients were converted to open surgery because of severe adhesions in the chest cavity. For patients with pleural effusion or those who need pulmonary surgery, preoperative evaluation was needed to determine the final procedure.

Approximately $35.8-52 \%$ of pediatric mediastinal masses occur in the posterior mediastinum, most of which are of neurogenic origin $(17,18)$. In addition, approximately $10-20 \%$ of posterior mediastinal neurogenic tumors have a spinal canal component (19). In our study, 54 (40\%) patients were diagnosed with posterior mediastinal tumors and 5 had intraspinal invasion. Since their tumors were suspected of neurogenic tumors, MRI was routinely performed to determine whether the tumor has intraspinal invasion. During the operation, a neurosurgeon was invited to participate in the surgery to ensure maximized removal of the tumor. One patient suffered local relapse and received reoperation. All those patients were alive and under close follow-up.

\section{Conclusions}

Our study had several limitations. Because we included 
all kind of mediastinal tumors, disease heterogeneity was inevitable. The proportion of cases with malignant and lager masses was higher in open surgery group than in VATS group. And other limitations include the small sample size, short follow-up, and retrospective nature. Despite those limitations, we describe our initial experience regarding the safety and effectiveness of VATS by comparing it to open surgery. VATS might be a safe and feasible approach for mediastinal tumors, and thoracotomy is appropriate for large tumors with neuroforaminal involvement. In the future study, multicentered and prospective investigations with a larger sample size and a longer follow-up are required to confirm the roles of these two procedures in pediatric patients.

\section{Acknowledgments}

Funding: This work was supported by funding from The National Natural Science Foundation of China (81770509), Jiangsu Provincial Special Program of Medical Science (BL2013003).

\section{Footnote}

Conflicts of Interest: The authors have no conflicts of interest to declare.

Ethical Statement: The authors are accountable for all aspects of the work in ensuring that questions related to the accuracy or integrity of any part of the work are appropriately investigated and resolved. This retrospective study was approved by the Medical Ethics Committee of Children's Hospital of Nanjing Medical University.

\section{References}

1. Rogers DA, Philippe PG, Lobe TE, et al. Thoracoscopy in children: An initial experience with an evolving technique. J Laparoendosc Surg 1992;2:7-14.

2. Kern JA, Rodgers BM. Thoracoscopy in the management of empyema in children. J Pediatr Surg 1993;28:1128-32.

3. Lacreuse I, Valla JS, de Lagausie P, et al. Thoracoscopic resection of neurogenic tumors in children. J Pediatr Surg 2007;42:1725-8.

4. Petty JK, Bensard DD, Partrick DA, et al. Resection of neurogenic tumors in children: Is thoracoscopy superior to thoracotomy? J Am Coll Surg 2006;203:699-703.
5. Fraga JC, Rothenberg S, Kiely E, et al. Video-assisted thoracic surgery resection for pediatric mediastinal neurogenic tumors. J Pediatr Surg 2012;47:1349-53.

6. Ng A, Bennett J, Bromley P, et al. Anaesthetic outcome and predictive risk factors in children with mediastinal tumours. Pediatr Blood Cancer 2007;48:160-4.

7. Lau G. Post-anaesthetic maternal death in a patient with mediastinal large b-cell lymphoma: A case report. Med Sci Law 2007;47:74-8.

8. Kawaguchi Y, Saito T, Mitsunaga T, et al. Prediction of respiratory collapse among pediatric patients with mediastinal tumors during induction of general anesthesia. J Pediatr Surg 2018;53:1365-8.

9. Said SM, Telesz BJ, Makdisi G, et al. Awake cardiopulmonary bypass to prevent hemodynamic collapse and loss of airway in a severely symptomatic patient with a mediastinal mass. Ann Thorac Surg 2014;98:e87-90.

10. Takeda S, Miyoshi S, Minami M, et al. Intrathoracic neurogenic tumors--50 years' experience in a japanese institution. Eur J Cardiothorac Surg 2004;26:807-12.

11. Ahmed G, Hegazy MM, Raafat T, et al. Surgical approaches, anaesthetic management and outcome in pediatric superior mediastinal tumors. J Egypt Natl Canc Inst 2017;29:147-53.

12. Rodgers BM, Talbert JL. Thoracoscopy for diagnosis of intrathoracic lesions in children. J Pediatr Surg 1976;11:703-8.

13. Dillon PW, Cilley RE, Krummel TM. Video-assisted thoracoscopic excision of intrathoracic masses in children: Report of two cases. Surg Laparosc Endosc. 1993;3:433-6.

14. Hwang SK, Park SI, Kim YH, et al. Clinical results of surgical resection of mediastinal teratoma: Efficacy of video-assisted thoracic surgery. Surg Endosc 2016;30:4065-8.

15. Liu HP, Yim AP, Wan J, et al. Thoracoscopic removal of intrathoracic neurogenic tumors: A combined chinese experience. Ann Surg 2000;232:187-90.

16. Li Y, Wang J. Experience of video-assisted thoracoscopic resection for posterior mediastinal neurogenic tumours: A retrospective analysis of 58 patients. ANZ J Surg 2013;83:664-8.

17. Takeda S, Miyoshi S, Akashi A, et al. Clinical spectrum of primary mediastinal tumors: A comparison of adult and pediatric populations at a single japanese institution. J Surg Oncol 2003;83:24-30. 
Page 8 of 8

18. Liu T, Al-Kzayer LFY, Xie X, et al. Mediastinal lesions across the age spectrum: A clinicopathological comparison between pediatric and adult patients. Oncotarget 2017;8:59845-53.

Cite this article as: Da M, Peng W, Mo X, Fan M, Wu K, Sun J, Qi J, Zhang Y. Comparison of efficacy between videoassisted thoracoscopic surgery and thoracotomy in children with mediastinal tumors: 6-year experience. Ann Transl Med 2019;7(22):653. doi: 10.21037/atm.2019.10.81

\section{Da et al. 6-year experience in children with mediastinal tumors}

19. Ozawa H, Kokubun S, Aizawa T, et al. Spinal dumbbell tumors: An analysis of a series of 118 cases. J Neurosurg Spine 2007;7:587-93. 